\title{
Estudo epidemiológico do câncer em menores de vinte anos, no estado de Sergipe- Brasil, no período de 1980-1999
}

Epidemiologic study of cancer in people under twenty years of age in the State of Sergipe, Brazil, from 1980 to 1999

Margareth Rose Uchoa Rangel,' Rosana Cipolotti, ${ }^{2}$ Amaury Lelis dal Fabbro, ${ }^{3}$

Anselmo Mariano Fontes ${ }^{4}$ e Márcio Botelho ${ }^{5}$

\section{Resumo}

Pouco se conhece sobre a epidemiologia das neoplasias malignas em crianças e adolescentes do estado de Sergipe. No presente trabalho apresentamos um estudo epidemiológico descritivo do câncer, neste estado brasileiro, em menores de 20 anos de idade, no período de 1980 a 1999.

M etodologia: foram revisados 1588 prontuários dos Serviços de O ncologia Pediátrica, sendo 233 do H ospital J oão Alves Filho e 1355 da Fundação de Beneficência H ospital de Cirurgia. D estes, foram selecionados 895 casos confirmados de câncer, com os quais realizamos 0 trabalho.

Resultados e conclusões: o grupo histológico que apresentou maior freqüência foi o dos Linfomas e N eoplasias do Sistema R etículo Endotelial. Q uando agrupamos todos os tumores, observamos que predominam os pacientes do sexo masculino (59,2\%), os pacientes considerados como não-brancos $(66 \%)$, que a topografia abdominal foi a mais freqüente $(26,7 \%)$, que a maioria dos sintomas teve início nos três meses que antecederam ao diagnóstico $(69,2 \%)$, a presença de uma tumoração foi a queixa inicial em $34,1 \%$ dos pacientes, a associação de cirurgia e quimioterapia foi a combinação terapêutica mais utilizada $(41,6 \%)$, o percentual de óbitos foi de $52,7 \%$, muitos pacientes abandonaram o tratamento $(19,4 \%)$ e que um pequeno número de pacientes foi considerado curado (12,1\%). A dificuldade de acesso aos serviços especializados, associada a fatores sócio-econômicos e a precária estruturação dos Serviços de O ncologia do Estado de Sergipe, colaboram para o diagnóstico tardio, estadiamento avançado e mau prognóstico.

Palavras-chave: neoplasias; estudos epidemiológicos; criança; adolescência; Brasil.

1Professora da disciplina de Cirurgia Pediátrica da Universidade Federal de Sergipe (UFS) e Cirurgiã nos dois Serviços de O ncologia Pediátrica de Sergipe. Enviar correspondência para M.R.U.R. Av. Gonçalo Rolemberg Leite 2399 / 1204; 49045-280 Aracaju, SE - Brasil. E-mail: mrrangel@uol.com.br

2Prof. D ra. da disciplina de pediatria da UFS e onco-hematologista do serviço de oncologia do H GJAF.

${ }^{3}$ Prof. Dr. da disciplina de epidemiologia da USP de Ribeirão Preto.

${ }^{4}$ Oncologista pediátrico e Chefe do serviço de oncologia do H GJAF.

${ }^{5}$ Cirurgião O ncológico e Chefe do Serviço de Oncologia da FBHC.

Recebido em agosto de 2001. 


\begin{abstract}
Introduction: very little is known about the malignant neoplasias that affect children and adoles cents in the State of Sergipe, Brazil. The present work shows a descriptive epidemiological study on cancer in this Brazilian state, in people younger than 20 years of age, from 1980 to 1999.

M ethodology: we reviewed 1588 cases from two clinics, 233 from the Hospital João Alves Filho and 1355 from the Fundação de Beneficência Surgical H ospital. From these, we selected 895 confirmed cases of malignant neoplasms in children and adolescents. They are the basis of our work.

Results: the most frequent histological types were lymphomas and neoplasias of the reticuloendothelial system. When all the tumors were grouped, we observed a predominance of male patients $(59,2 \%)$; a predominance of patients considered non-white $(66,0 \%)$; abdominal topography was the most common $(26,7 \%)$; the symptoms started 3 months prior to the diagnosis $(69,2 \%)$; the presence of a swelling was the initial complaint $(34,1 \%)$; the association of surgery and chemotherapy was the most used therapeutic combination $(41,6 \%)$; the percentage of death was $52,7 \%$; $19,4 \%$ of the patients abandoned treatment, and $12,1 \%$ of patients were considered cured.

Conclusions: the lack of specialized services available associated with socio-economic factors, and the precarious infrastructure of the State of Sergipe's oncology services are in part responsible for the late diagnosis, advanced stage of the disease and poor prognosis.
\end{abstract}

Key words neoplasms, epidemiological studies; child; adolescence; Brazil.

\section{INTRODUÇÃO}

Apesar de corresponder a apenas $2 \%$ a $3 \%$ de todos os diagnósticos de câncer, as neoplasias malignas na infância representam nos países desenvolvidos a segunda causa de morte entre 0-14 anos de idade. ${ }^{1} \mathrm{~N}$ os países em desenvolvimento, representam a terceira causa de morte. No estado de São Paulo, no grupo compreendido entre 5 e 14 anos, já éa primeira causa de morte, excluindo-se as causas externas. ${ }^{2}$

A incidência do câncer na infância é de 100 a 150 casos por milhão de habitantes por ano e aumentou cerca de $12 \%$ nos últimos 15 anos. A incidência específica varia de acordo com o tipo de câncer e com o país ou a região que se estude. ${ }^{1}$ Considerando-se que a população brasileira tem cerca de $50 \%$ de habitantes abaixo de 20 anos de idade, podemos estimar em torno de 11.0000 número de novos casos de câncer por ano em todo 0 país, nesta faixa etária. ${ }^{2}$ Combinando-se todos os tipos de câncer na infância e adolescência, observou-se que a incidência foi geralmente maior para meninos do que para meninas, e em brancos do que em negros da mesma idade. ${ }^{1,3}$

0 câncer na infância geralmente tem progressão rápida, porém silenciosa e oligossintomática. Entre os sintomas mais freqüentes estão: palidez cutâneo-mucosa, adenomegalia, visceromegalias, cefaléia, vômitos, massa abdominal ou aumento do volume em partes moles, dor óssea ou articular, febre, emagrecimento e sangramento (petéquias, hematúria, epistaxe ou sangramento gengival).4-6

A mortalidade por câncer na infância diminuiu substancialmente na última década. N os EU A a mortalidade total foi reduzida em $40 \%$ e no Canadá em $50 \%$ para todas as idades e ambos os sexos. $N$ os países latinoamericanos esta avaliação é dificultada pela falta de dados. ${ }^{2,6,7}$

\section{MATERIAL E MÉTODO}

Os registros hospitalares dos Serviços de O ncologia Pediátrica dos H ospitaisJ oão Alves Filho e Fundação de Beneficência H ospital de Cirurgia, Aracaju-SE, foram utilizadas como fontes de informação. Foram revisados 1588 prontuários, dos quais 895 tinham diagnósticos de neoplasias malignas, com os quais realizamos 0 trabalho. Analisamos características de sexo, idade, raça, procedência, diagnóstico histopatológico, sintomatologiainicial, tempo de aparecimento 
dos primeiros sintomas, topografia, tratamento e evolução clínica. Elaboramos um banco de dados no programa M icrosoft Access e posteriormente 0 transferimos para 0 programa Epilnfo versão 6.0 , onde foi realizada a análise estatística.

\section{RESULTADOS}

As cidades de Sergipe que apresentaram maior número de diagnóstico de câncer foram Aracaju com 253 casos (27,4\%), I tabaiana com $54(5,9 \%)$, Lagarto com 42 (4,6\%), N ossa Senhora do Socorro com 39 (4,2\%), São Cristóvão com 23 casos (2,5\%), e Estância com 19 casos (2,1\%), correspondendo juntas a $48,0 \%$ dos casos. Estas cidades são também as mais populosas do estado (49,8\% da população) e também estão situadas próximas à capital onde estão localizados os dois serviços de oncologia do estado. (Figura 1)

O bservamos predomínio dos pacientes não-brancos, com 593 casos (66,2\%), o que reflete as características gerais da população do estado de Sergipe e de pacientes do sexo masculino com 530 casos $(59,2 \%)$, quando agrupados todos os tumores e faixas etárias.

0 tipo histológico mais freqüente foi 0 grupo dos linfomas e neoplasias do sistema retículo endotelial (NRE), com 254 casos $(28,4 \%)$, seguido pelo grupo das leucemias e tumores renais. (Figura 2)

Em todas as faixas etárias houve predomínio dos tumores hematológicos sobre os tumores sólidos. As neoplasias renais, do sistema nervoso simpático, retinoblastoma e neoplasias hepáticas foram mais freqüentes no grupo etário de 0-4 anos. 0 s tumores do SN C predominaram na faixa etária de 5-9 anos. $\mathrm{N}$ a faixa dos 10 aos 14 anos aumentou a freqüência dos tumores de células germinativas e trofoblásticas e dos tumores ósseos. No grupo dos maiores de 15 anos cresceram ainda mais os tumores ósseos. Os carcinomas e as neoplasias epiteliais, extremamente raros nos menores de cinco anos, aumentaram em freqüência com a idade, chegando a 8\% nos maiores de 15 anos. Os sarcomas de partes moles apresentaram uma maior freqüência nos menores de cinco anos e depois se mantiveram relativamente constantes em todos os grupos etários. (Tabela 1)

A combinação terapêutica mais freqüente foi a de cirurgia com quimioterapia em 372 casos (41,6\%), 196 pacientes usaram apenas quimioterapia (21,9\%), 135 receberam tratamento com cirurgia, quimio e radioterapia $(15,1 \%)$.

Figura 1. Casos de câncer segundo municípios de residência, Estado de Sergipe, 1980 a 1999

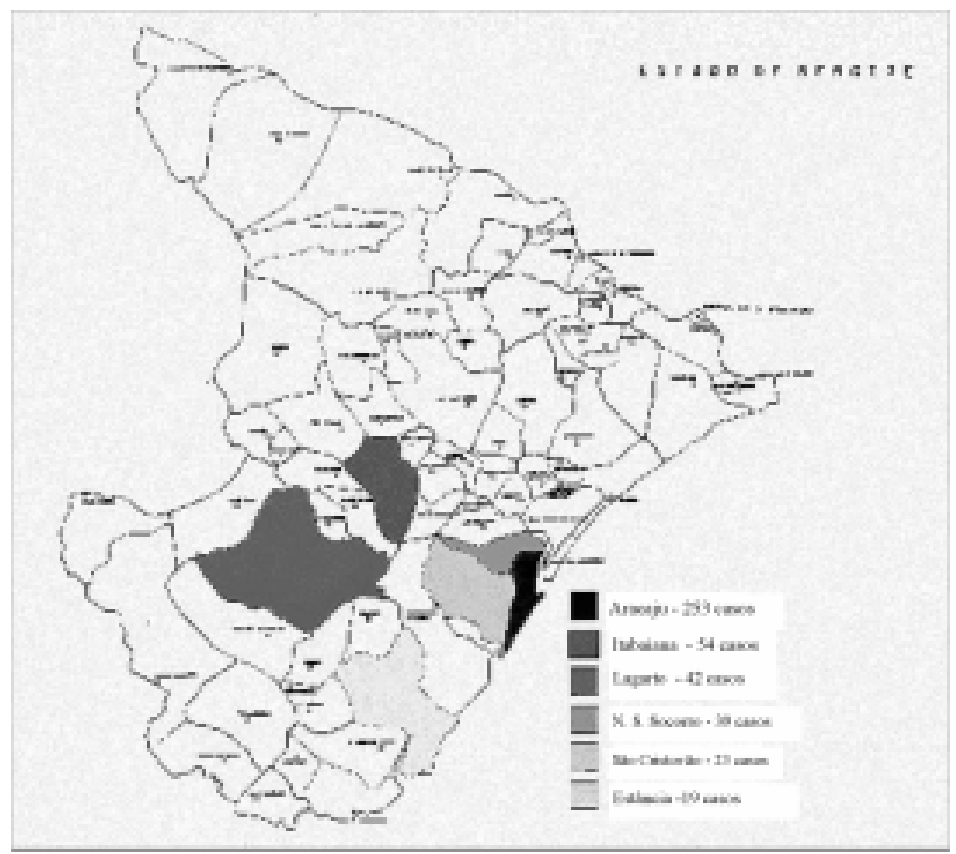

Figura 2. Distribuição dos tumores pediátricos por tipo histológico em Aracaju (SE) 1980 a 1999

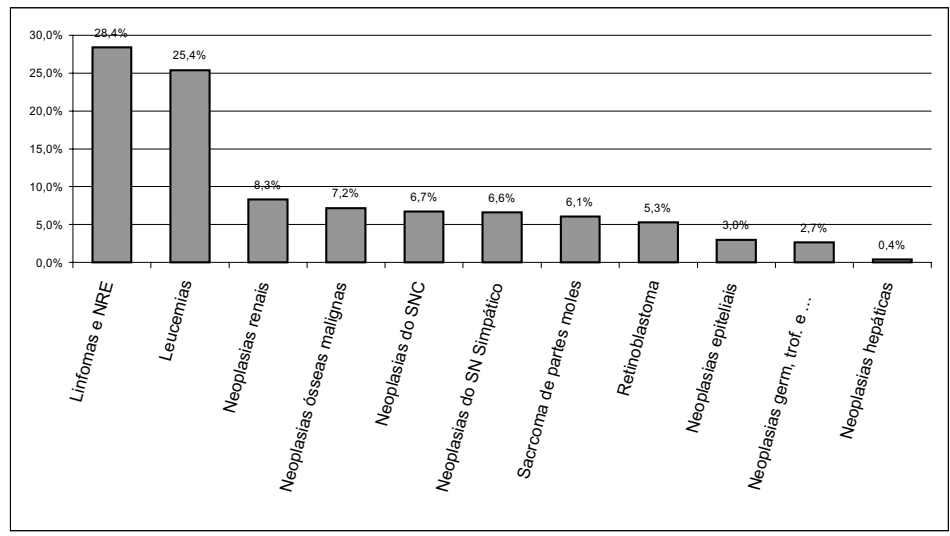

A topografia mais encontrada foi a abdominal com 239 casos $(26,7 \%)$, principalmente as custas dos linfomas com infiltração intestinal, tumores renais e neuroblastomas. Em segundo lugar encontramos a 
medula óssea com 237 casos $(26,5 \%)$ e em terceiro a cabeça e pescoço com 156 casos $(17,5 \%)$ onde também predominaram os linfomas.

0 sintoma mais freqüente foi a presença de tumoração em 336 casos (34,1\%), seguido pela palidez em 187 casos (19\%) e dor em 122 casos (12,4\%). 0 tempo entre o início dos sintomas e o diagnóstico variou amplamente. $\mathrm{N}$ a maioria dos casos $(69,1 \%)$ os sintomas iniciaram nos três meses que antecederam o diagnóstico.

Encontramos um grande número de óbitos, 472 casos $(52,7 \%)$, assim como uma taxa de abandono de tratamento também bastante expressiva, 174 casos $(19,4 \%)$. Apenas 108 pacientes $(12,1 \%)$ foram considerados curados após cinco anos do término do tratamento e sem evidência de doença maligna.

O bserva-se que o percentual de óbitos reduziu significativamente nos últimos anos, passando de $61,9 \%$ nos anos de 80-84 para 39,4\% após 1995. 0 índice de abandono também caiu de $18,7 \%$ para 13,2\%. Grande parte dos pacientes matriculados após 1995 ainda se encontravam em tratamento ou em controle $(47,3 \%)$ podendo haver também uma melhora no percentual de cura, muito baixo até 1994 (17,9\%). (Figura 3)

\section{DISCUSSÃO}

No estado de Sergipe observamos que as cidades que apresentam maiores freqüências de câncer em crianças e adolescentes são também as mais populosas. $\mathrm{N}$ otamos ainda que estão localizadas próximas da capital onde se encontram os dois Serviços de O ncologia do Estado, sugerindo que a proximidade e a facilidade de acesso aos serviços especializados sejam fatores determinantes para este evento e que exista sub-registro nas cidades mais afastadas. O bservamos neste estudo que os pacientes considerados não-brancos representam $66,2 \%$ de todos os casos e relacionamos este achado ao fato de dois terços da população sergipana ser considerada não-branca (305.856 habitantes), segundo dados do Censo do IBGE de 1996. ${ }^{10}$

Assim como na literatura internacional, observamos em Sergipe o predomínio dos tumores hematológicos $(53,8 \%) .{ }^{11}$ Porém, quando analisamos os tumores por tipos histológicos, o grupo dos linfomas eneoplasias do sistema retículo endotelial ocupam 0 primeiro lugar em freqüência entre todas as neoplasias malignas $(28,4 \%)$ e no grupo dos linfomas predominam o tipo não $\mathrm{H}$ odgkin, cuja topografia abdominal foi a mais

Figura 3. Evolução por grupo de cinco anos dos pacientes oncológicos pediátricos em Aracaju (SE), 1980 a 1999.

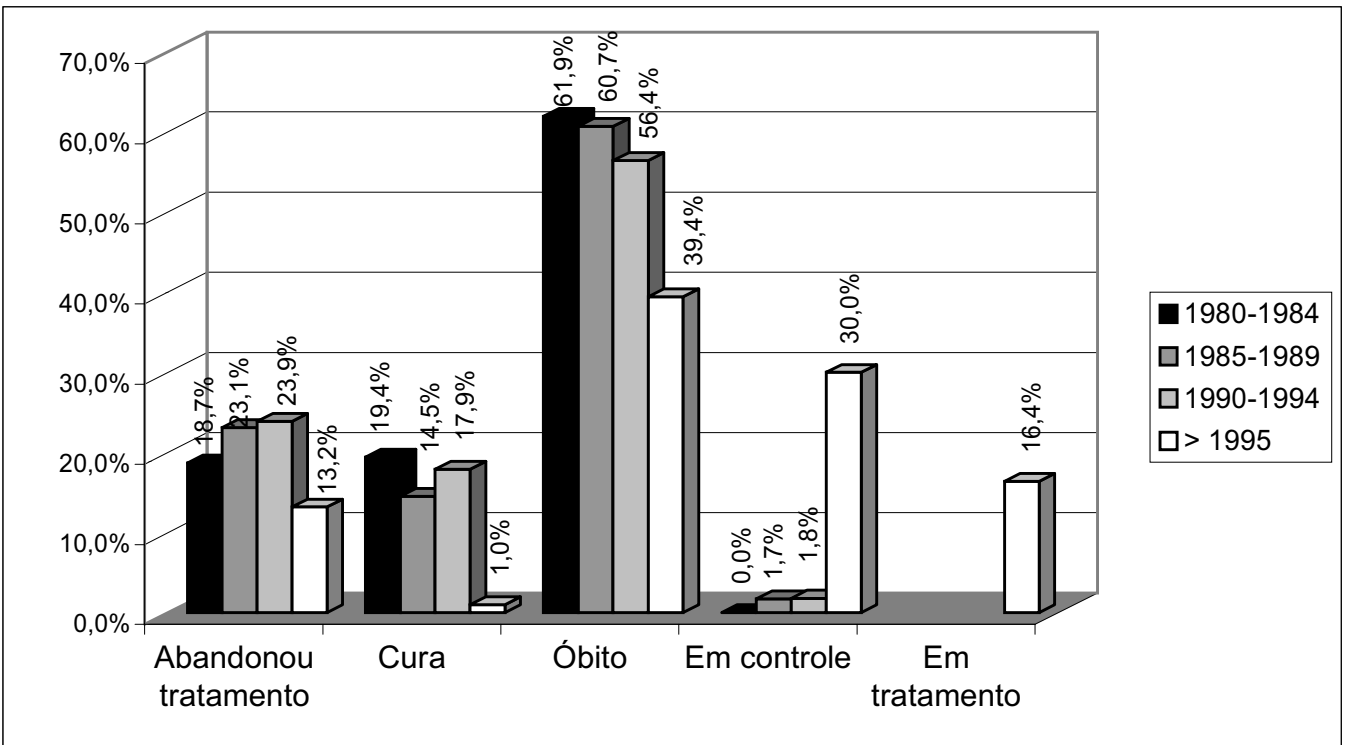


Tabela 1. Distribuição dos tumores por tipo histológico x grupo etário em Aracaju (SE) de 1980 a 1999

\begin{tabular}{|c|c|c|c|c|c|c|c|c|c|c|}
\hline \multirow{2}{*}{$\begin{array}{l}\text { Grupo etário } \\
\text { Tipo de tumor }\end{array}$} & \multicolumn{2}{|c|}{0 a 4 anos } & \multicolumn{2}{|c|}{5 a 9 anos } & \multicolumn{2}{|c|}{10 a 14 anos } & \multicolumn{2}{|c|}{15 a 19 anos } & \multicolumn{2}{|c|}{ Total } \\
\hline & $\mathrm{n}$ & $\%$ & $\mathrm{n}$ & $\%$ & $n$ & $\%$ & $n$ & $\%$ & $\mathrm{n}$ & $\%$ \\
\hline Leucemias & 82 & 21,5 & 68 & 28,2 & 61 & 30,8 & 16 & 21,3 & 227 & 25,4 \\
\hline Linfomas e NRE & 93 & 24,4 & 84 & 34,9 & 53 & 26,8 & 24 & 32,0 & 254 & 28,4 \\
\hline $\begin{array}{l}\text { Neop. germ, trofo e } \\
\text { gonadais. }\end{array}$ & 4 & 1,0 & 8 & 3,3 & 10 & 5,1 & 2 & 2,7 & 24 & 2,7 \\
\hline Neoplasias do SNS & 44 & 11,5 & 11 & 4,6 & 3 & 1,5 & 1 & 1,3 & 59 & 6,6 \\
\hline Neoplasias do SNC & 22 & 5,8 & 20 & 8,3 & 14 & 7,1 & 4 & 5,3 & 60 & 6,7 \\
\hline Neoplasias epiteliais & 3 & 0,8 & 7 & 2,9 & 11 & 5,6 & 6 & 8,0 & 27 & 3,0 \\
\hline Neoplasias ósseas & 5 & 1,3 & 8 & 3,3 & 34 & 17,2 & 17 & 22,7 & 74 & 7,2 \\
\hline Neoplasias renais & 56 & 14,7 & 17 & 7,1 & 1 & 0,5 & & & 64 & 8,3 \\
\hline Retinoblastoma & 42 & 11,0 & 3 & 1,2 & 1 & 0,5 & 1 & 1,3 & 47 & 5,3 \\
\hline Sarcoma de partes moles & 26 & 6,8 & 15 & 6,2 & 10 & 5,1 & 4 & 5,3 & 55 & 6,1 \\
\hline Neoplasias hepáticas & 4 & 1 & - & - & - & - & - & - & 4 & 0,4 \\
\hline TOTAL & 381 & 100,0 & 241 & 100,0 & 198 & 100,0 & 75 & 100,0 & 895 & 100,0 \\
\hline
\end{tabular}

encontrada $(40,6 \%)$. Se associarmos os linfomasnão $\mathrm{H}$ odgkin aos deBurkitt, teremos $66,9 \%$ deste grupo de neoplasias. O s linfomas também ocupam o primeiro lugar em freqüência, entre os tumores em menores de 20 anos na Nigéria (59\%) e no Paquistão (26\%). É possível que esta casuística observada neste estudo esteja relacionada com a etnia da população. ${ }^{12,13}$

$\mathrm{N}$ as duas últimas décadas, com 0 avanço tecnológico, o número de diagnóstico de tumores do SNC aumentou consideravelmente. Países com sistemas de saúde bem estruturados têm os maiores coeficientes de incidência. ${ }^{14} \mathrm{~N}$ este estudo os tumores do SN C ocupam o quinto lugar em freqüência, a partir da década de 90 houve um aumento no número de diagnósticos de tumores do SN C, correspondendo ao período em que foi instalado o serviço de tomografia computadorizada no estado de Sergipe. Este recurso tecnológico, sem dúvida, colaborou para que um número maior de diagnósticos fosse realizado. Antes disso, provavelmente muitos pacientes ficaram sem confirmação diagnóstica e morreram sem acesso ao tratamento adequado.

N os Serviços de O ncologia do Estado de Sergipe observam-se problemas financeiros e estruturais importantes, que levam à descontinuidade do tratamento quimioterápico, seja por falta de drogas ou por problemas sociais dos pacientes, que freqüentam irregularmente os serviços. Encontramos um percentual de óbitos muito elevado (52,7\%), enquanto que apenas $12 \%$ dos pacientes foram considerados curados. Estes resultados estão muito aquém da média de estatísticas nacionais e internacionais. Porém, se observarmos ao longo dos anos, veremos que estenúmero caiu de $61,9 \%$ nos anos de 1980 a 1984, para $39,4 \%$ de 1995 a 1999. Consideramos que alguns fatores possam estar influenciando estes resultados negativos encontrados em nossos serviços, como a precariedade de instalações, a limitação no número de leitos, a falta de continuidade no tratamento, as condições nutricionais dos pacientes, a falta de isolamentos e de leitos em unidade de terapia intensiva para dar suporte aos pacientes nas fases de neutropenia e/ou pancitopenia, durante 0 tratamento quimioterápico. Acreditamos que fatores sócio-econômicos estejam diretamente relacionados com os casos de abandono.

\section{CONCLUSÕES}

0 grupo histológico que apresentou maior freqüência foi o dos linfomas e neoplasias do sistema retículo endotelial. Quando agrupamos todos os tumores, observamos que predominam os pacientes do sexo masculino $(59,2 \%)$, dos pacientes considerados como não-brancos (66\%), que a topografia abdominal foi a mais freqüente $(26,7 \%)$, que a maioria dos sintomas iniciaram nos três meses que antecederam ao diagnóstico $(69,2 \%)$, a presença de uma tumoração foi a queixa inicial em $34,1 \%$ dos pacientes, a associação de cirurgia e quimioterapia foi a 
combinação terapêutica mais utilizada (41,6\%), o percentual de óbitosfoi de $52,7 \%$, muitos pacientes abandonaram o tratamento $(19,4 \%)$ e que um número pequeno de pacientes foram considerados curados (12,1\%).

Para que possamos produzir estudos epidemiológicos sobre o câncer em crianças énecessário estabelecer uma padronização dos dados nos prontuários e a implantação dos registros de base populacional bem caracterizado e com alto nível de confiança.

D evido à complexidade de se estudar um número tão grande de tumores, com características tão diversas, julgamos necessário realizarmos estudos isolados dos diferentes grupos de tumores.

Faz-se necessária uma reestruturação dos Serviços de 0 ncologia Pediátrica de Sergipe, para que possamos oferecer maiores recursos diagnósticos e tratamentos adequados às crianças e adolescentes portadores de câncer. Só assim poderão ser obtidos melhores resultados.

\section{REFERÊNCIAS BIBLIOGRÁFICAS}

1. Ries LAG, Smith M A, Gurney JG, Linet M , TamraT, Young $L$, et al. Cancer incidenceand survival among children and adolescents. U nited States SEER Program 1975-1995. 1999;99:182.

2. Petrilli AS, Carneiro J L Jr, Cypriano M , Angel A, Toledo S. D iferençasdínicas, epidemiológicas ebiológicas entrecâncer na criança eno adulto. Rev Bras C ancerol 1997;43(3):191-203.

3. Bleyer WA. M agnitude of the childhood cancer problem. Cancer Bull 1992;44:444-50.

4. H elman L, Thiele CJ. N ew insights into the causes of cancer. Pediatr Clin N orth Am
1991;38:201.

5. N arod SA. G enetic epidemiology of childhood cancer. Biochim Biophys Acta 1996; 141-50.

6. Sarapião CJ 0 câncer nainfância. Ars Curandi 1988;7:96-102.

7. Youg G, Toretsky JA, C ampbell AB, Eskenazi AE. Recognition of common childhood malignancies. Am Fam Physician 2000;61(7):214454.

8. H udson M M , Jones D, Boyett J, Sharp GB, Pui CH . Latemortality of long-term survivors of childhood cancer. J Clin O ncol 1997;15(6):2205-13.

9. Linet MS, Ries LA, Smith MA, Tarone RE, D evassa SS. C ancer Surveillance series: recent trends in childhood cancer incidenceand mortality in the U nited States. J N atl C ancer Inst 1999;91(12):1051-8.

10. Instituto Brasileiro de G eografia eEstatística. Resultados da amostra do censo demográfico deSergipe. Censo demográfico. Rio deJ aneiro: Fundação IBGE; 1996. p.16-21.

11. Fajado-G utierrez $A$, M ejia-Arangure J $M$, $\mathrm{H}$ ermanez-Cruz $\mathrm{L}$, M endonza-Sanchez $\mathrm{H} \mathrm{F}$, Garduno-Espinosa J, M artinez-Garcia M C. Epidemiologia descriptiva de las neoplasias malignas en niños. Pan Am J Public H ealth 1999;6(2):75-88.

12. Stiller CA, Parkin D M . G eographic and ethnic variations in theincidence of childhood cancer. Br M ed Bull 1996;52:682-703.

13. Stiller CA, Bunch KJ, Lewis JJ. Ethnic group and survival from childhood cancer: report from the U K C hildren's C ancer Study G roup. Br J Cancer 2000;82(7):1339-43.

14. Glass AG , Fraumeni JF. Epidemiology of bone cancer in children. J N atl Cancer Inst 1970;44:187.

15. Bleyer WA. W hat can belearned about childhood cancer from cancer statistic: review 19731978. C ancer 1993;71:3229-36. 